\title{
Gender-Specific Association between Alcohol Consumption and Stress Perception, Depressed Mood, and Suicidal Ideation: The 2010-2015 KNHANES
}

\author{
Jo-Eun Jeong ${ }^{1}$, Soo-Hyun $\mathrm{Joo}^{2}$, Changtae Hahn', Dai-Jin Kim², and Tae-Suk Kim² ${ }^{凶}$ \\ 'Department of Psychiatry, Daejeon St. Mary's Hospital, College of Medicine, The Catholic University of Korea, Daejeon, Republic of Korea \\ ${ }^{2}$ Department of Psychiatry, Seoul St. Mary's Hospital, College of Medicine, The Catholic University of Korea, Seoul, Republic of Korea
}

\begin{abstract}
Objective Alcohol drinking can cause various psychiatric and medical diseases. Although women generally consume less alcohol than men, they may be at a greater risk for alcohol-related psychological distress. The aim of the current study is to evaluate whether the association between alcohol consumption and psychological distress, including stress, depressed mood, and suicidal ideation and the risks of psychological distress differ based on gender.

Methods The cross-sectional study included 31,657 participants (17,915 women and 13,742 men) from the 2010-2013 and 2015 Korea National Health and Nutrition Examination Survey. Alcohol drinking and Alcohol Use Disorders Identification Test (AUDIT) levels were assessed for evaluating the amount of alcohol intake and alcohol-related problems. Self-perception of stress, depressed mood, and suicidal ideation were assessed for evaluating psychological distress. Odds ratio and $95 \%$ confidence intervals for psychological distress were calculated using multiple logistic regression models.

Results The risks of psychological distress were not significantly associated with drinking level in both sexes. However, the risks of psychological distress were associated with an increase in AUDIT levels, and there were gender differences in the psychological consequences of alcohol-related problems. The association was more drastic in women, and women showed a significant association even though the severity of drinking problem was low with the exception of stress perception.

Conclusion The risks of psychological distress were associated with the severity of alcohol-related problems and women were more likely to be susceptible. Therefore, it is recommended that women even at low-risk for problematic drinking should be screened for psychological distress.

Psychiatry Investig 2019;16(5):386-396
\end{abstract}

Key Words Alcohol drinking, Stress, Psychological, Depression, Suicidal ideation, Sex.

\section{INTRODUCTION}

Alcohol is consumed worldwide, and drinking alcohol is a common part of many cultures. The World Health Organization (WHO) 2014 global status report on alcohol and health revealed that individuals above the age of 15 drink 6.2 liters of pure alcohol per year on average, which translates into 13.5 grams of pure alcohol per day. ${ }^{1}$ In South Korea, the rate of cur-

Received: November 23, 2018 Revised: February 18, 2019 Accepted: February 28, 2019

$\triangle$ Correspondence: Tae-Suk Kim, MD, PhD

Department of Psychiatry, Seoul St. Mary's Hospital, College of Medicine, The Catholic University of Korea, 222 Banpo-daero, Seocho-gu, Seoul 06591, Republic of Korea

Tel: +82-2-2258-6085, Fax: +82-2-594-3870, E-mail: bluenote@catholic.ac.kr (a) This is an Open Access article distributed under the terms of the Creative Commons Attribution Non-Commercial License (https://creativecommons.org/licenses/bync/4.0) which permits unrestricted non-commercial use, distribution, and reproduction in any medium, provided the original work is properly cited. rent drinkers has been steadily increasing from $72.6 \%$ in 2005 to $75.3 \%$ in 2016 among men and from $36.9 \%$ in 2005 to $48.9 \%$ in 2016 among women. ${ }^{2}$ In particular, the rate of high-risk drinking (more than 5 drinks for women and more than 7 drinks for men on single drinking occasions and drinking more than twice a week) has risen twice as much in women, from $3.4 \%$ in 2005 to $6.3 \%$ in $2016 .^{2}$

Alcohol consumption is associated with not only medical diseases, but also mental health. ${ }^{1}$ The data from the National Epidemiologic Survey on Alcohol and Related Conditions on alcohol consumption and medical conditions reveal that approximately $25 \%$ to $30 \%$ of alcohol users have health problems, independent of the quantity consumed. ${ }^{3}$

In addition, alcohol is a psychotropic central nervous system (CNS) depressant that interferes with the brain's communication pathways implicated in psychological symptoms. ${ }^{4}$ 
Previous studies have indicated that increased exposure to alcohol increases risk of depression ${ }^{5,6}$ and alcohol can serve as a stressor itself. ${ }^{7}$ Moreover, alcohol drinking acts as an important risk factor for attempted or completed suicide, even among individuals who are not alcoholics. ${ }^{8}$

Alcohol-related harm may be determined by the volume of alcohol consumed, but is also influenced by the pattern of drinking or the quality of alcohol consumed. ${ }^{9,10}$ Previous studies have revealed that irregular heavy drinking plays a crucial role in determining the outcome in the case of cardiovascular disorders. ${ }^{3}$

Furthermore, previous studies have showed gender differences in the effects of drinking alcohol and alcohol-related problems. ${ }^{11-14}$ Several studies have indicated that females experience an earlier onset of adverse somatic consequences of alcohol use ("telescoping effect"). The telescoping effect indicated that women are more vulnerable to alcohol consumption. ${ }^{15}$ Women also appear to be more vulnerable to the neurotoxic effects of alcohol than men although women generally consume less alcohol than men. ${ }^{11}$ Similarly, there are some differences in the influence on psychological distress between males and females. ${ }^{12}$ Compared with men, alcohol-dependent women more often have comorbid mood and anxiety disorders and increased intensity of depressive symptoms. ${ }^{13}$ Women who are alcoholics are known to have a five-fold higher suicide rate than those without alcoholism, which is higher than the rate in men with alcoholism. ${ }^{13}$

However, the majority of clinical and experimental approaches about the effects of alcohol and its consequences have been focused on male participants, and comparative studies of men and women have mainly focused on the physical effect of alcohol and patients with alcohol use disorders (AUD) rather than the general population.

Therefore, the present study assesses how the association between alcohol consumption and psychological distress differs across gender in a large sample of a Korea population using standard and nationally representative data from the Korea National Health and Nutrition Examination Survey (KNHANES). Based on previous findings, we hypothesized the following: 1) psychological distress is more likely to occur when there is more alcohol consumption and problematic drinking; and 2) in women, the influence of alcohol consumption and problematic drinking on psychological distress is more severe than in men.

\section{METHODS}

\section{Data source and study sample}

This study was based on data obtained from the fifth and sixth KNHANES conducted by the Korea Centers for Disease
Control and Prevention in 2010-2012 (5th KNHANES) and 2013-2015 (6th KNHANES), respectively. We used the data from 2013 and 2015 except 2014 from the 6th KNHANES because some of the items in the alcohol use disorders identification test (AUDIT) were not investigated in 2014.

The KNHANES is a nationwide population-based survey of the health and nutritional status of Korean people. Annually approximately 8,000-10,000 individuals from about 4,000 households are selected to represent noninstitutionalized Koreans using a stratified and multi-stage probability sampling design with a rolling survey-sampling model. The sampling frames were based on the 2009 resident registration population and the 2008 data of the apartment market for the 5th KNHANES and 2010 population and housing consensus for the 6th KNHANES. The survey was composed of three parts: a health interview, a health examination, and a nutrition survey. Trained interviewers and medical technicians conducted interviews using a structured questionnaire. Physical examination, blood sampling, and urine sampling were performed at a mobile examination center. We used only health-related data, and all items of health-related data were obtained using faceto-face interviews.

The response rates were $80.8 \%(n=25,534)$ in the 5 th and $78.3 \%(n=22,948)$ in the 6 th KNHANES. Data from a total of 31,657 participants (17,915 women and 13,742 men) who were 19 years of age or older were analyzed for this study (Figure 1).

\section{Sociodemographic and health-related data}

Sex, age, years of education, marital status, employment status, household income, smoking status, physical activity status, sleep duration, and presence of medical or psychiatric conditions were used.

The years of education were categorized as $\leq 6$ years (elementary), 7-9 years (middle school), 10-12 years (high school), and $\geq 13$ years (college). Marital status was classified as single, married, or others (divorced, separated, or widowed). Employment status was divided into working and not working. Household income was categorized according to quartiles of total income for each member in the household. Smoking status was defined as current smoker, ex-smoker, or never smoker. Regular exercise was defined as physical activity performed for a minimum of 20 minutes thrice a week. All the participants were asked to assess sleep duration: "How long do you usually sleep every night?" The presence of medical or psychiatric conditions was diagnosed by a doctor, which was dichotomized into present (at least one) and none (Table 1). In women, the menstruation condition was also assessed whether they were menstruating or were amenorrheic due to pregnancy, lactation, or menopause. 


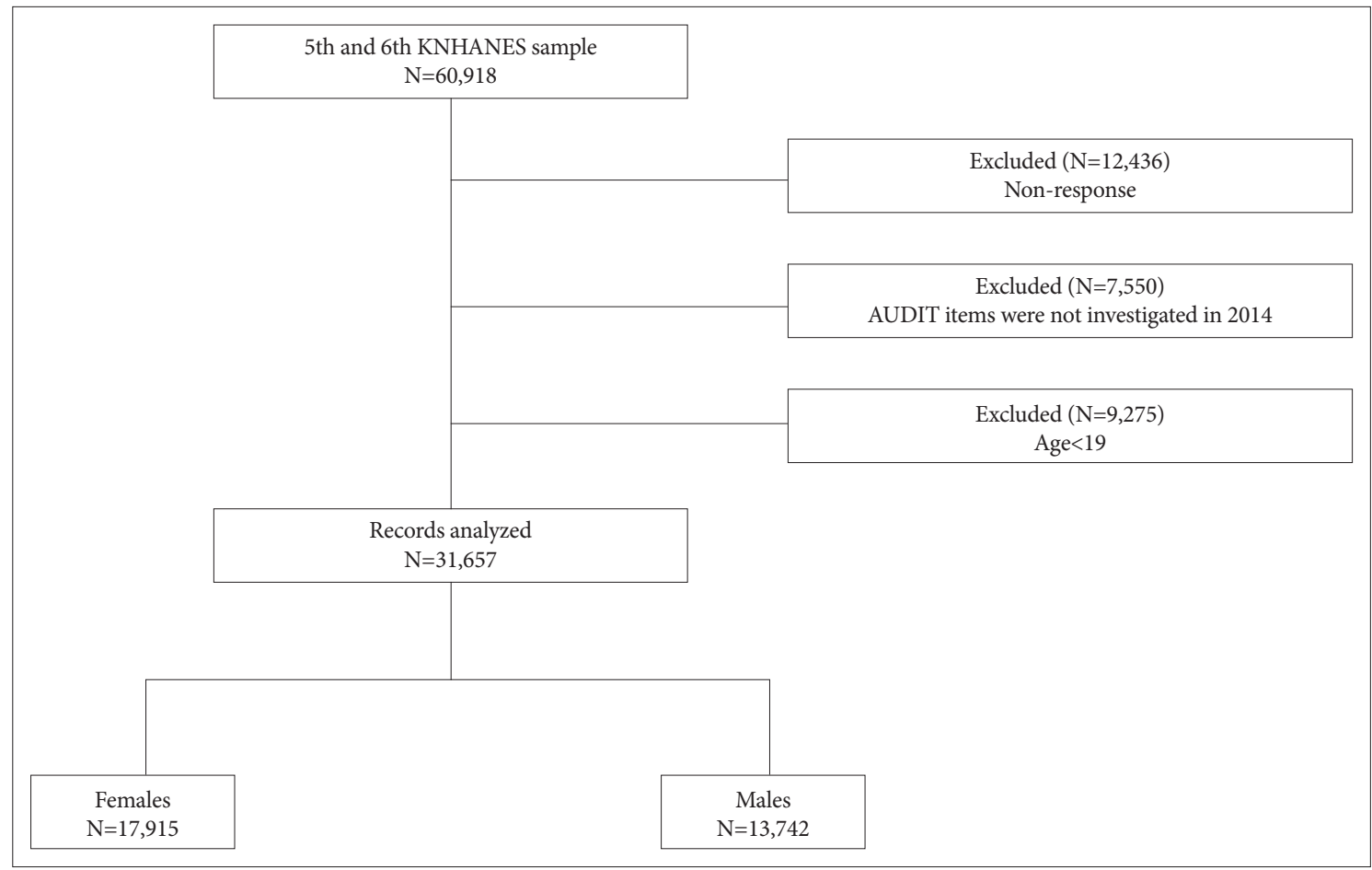

Figure 1. Flow diagram of the study participants. KNHANES: Korea National Health and Nutrition Examination Survey, N: numbers, AUDIT: Alcohol Use Disorders Identification Test.

\section{Measurement of alcohol-related variables}

Alcohol drinking and AUDIT levels were assessed for evaluating the participants' amount of alcohol intake and alcoholrelated problems.

To assess the drinking level, the amount of pure alcohol consumed (in grams per day) was calculated using the average number of alcoholic beverages consumed and the frequency of alcohol consumption. Additionally, the participants were classified into three groups according to the amount of alcohol consumption per day: non-drinker ( $<1 \mathrm{~g} /$ day), mild to moderate drinker (1-30 g/day), and a heavy drinker $(>30$ g/day). ${ }^{16}$

In addition, participants were also divided into four groups depending on the AUDIT scores: $0-7,8-15,16-19, \geq 20$. AUDIT is a widely used 10-item screening instrument developed by the WHO for assessing AUD. This test comprehensively evaluates the multidimensional aspects of alcohol consumption over the last year such as risky or harmful drinking pattern including alcohol dependence and problems related to alcohol consumption, not limited to the volume and frequency of alcohol intake. ${ }^{17}$ The total scores range from 0 to 40 , with the higher scores indicating a greater tendency towards problematic drinking or AUD and the need for an intensive intervention. According to WHO guidelines for AUDIT use, the scores of 0-7 are classified as Zone 1 (low-risk drinking, intervention includes alcohol education), 8-15 as Zone 2 (hazardous drinking, intervention with simple advice), 16-19 as Zone
3 (harmful drinking, with simple advice plus brief counseling and continued monitoring), and more than 20 as Zone 4 (possible alcohol dependence, with referral to a specialist setting for diagnostic evaluation and treatment). ${ }^{18,19}$ High-risk drinking was defined as drinking $>60 \mathrm{~g}$ pure alcohol per drinking day for men and $>40 \mathrm{~g}$ per drinking day for women for two or more times a week. ${ }^{20}$

\section{Measurement of psychological distress}

Stress perception, depressed mood, and suicidal ideation were assessed for evaluating the participants' psychological distress.

Stress perception was assessed using the following question: "How stressed are you on a daily basis?" "Extremely stressed" or "quite stressed" responses were classified as stress perception, and "a little bit stressed" and "hardly stressed" were classified as no stress perception.

To assess depressed mood, participants answered "yes" or "no" in response to whether they had experienced a depressed mood for two or more continuous weeks during the previous year. "During the past year, have you felt feelings of sadness or hopelessness that persisted for at least two weeks and that disrupted your social life?" This question is included in the WHO Composite International Diagnostic Interview-Short Form, which was validated as a cost-effective screening method for a general public survey. ${ }^{21} \mathrm{~A}$ "yes" or "no" response was used to determine whether the participants had experienced depressed 
Table 1. Demographic characteristics according to gender

\begin{tabular}{|c|c|c|c|}
\hline Variables & Female & Male & $\mathrm{p}$ value \\
\hline Age (years) $(\mathrm{N}=29,995)$ & $46.8 \pm 0.2$ & $44.8 \pm 0.2$ & $<0.001$ \\
\hline Education $(\mathrm{N}=28,294)$ & $16,283(100.0)$ & $12,011(100.0)$ & $<0.001$ \\
\hline$\leq$ Elementary & $4,994(30.7)$ & $2,080(17.3)$ & \\
\hline Middle school & $1,679(10.3)$ & $1,367(11.4)$ & \\
\hline High school & $5,148(31.6)$ & $4,372(36.4)$ & \\
\hline$\geq$ College & $4,462(27.4)$ & $4,192(34.9)$ & \\
\hline Occupation $(\mathrm{N}=28,239)$ & $16,287(100.0)$ & $11,952(100.0)$ & $<0.001$ \\
\hline Yes & $7,764(47.7)$ & $8,785(73.5)$ & \\
\hline No & $8,523(52.3)$ & $3,167(26.5)$ & \\
\hline Household income $(\mathrm{N}=29,649)$ & $16,949(100.0)$ & $12,700(100.0)$ & $<0.001$ \\
\hline Low & $3,620(21.3)$ & $2,308(18.2)$ & \\
\hline Moderate-low & $4,365(25.8)$ & $3,254(25.6)$ & \\
\hline Moderate-high & $4,422(26.1)$ & $3,510(27.6)$ & \\
\hline High & $4,542(26.8)$ & $3,628(28.6)$ & \\
\hline Marital status $(\mathrm{N}=29,939)$ & $17,121(100.0)$ & $12,818(100.0)$ & $<0.001$ \\
\hline Single & $2,139(12.5)$ & $2,171(16.9)$ & \\
\hline Married & $11,630(67.9)$ & $9,924(77.4)$ & \\
\hline Others & $3,352(19.6)$ & $723(5.7)$ & \\
\hline Drinking levels $(\mathrm{N}=24,652)$ & $13,094(100.0)$ & $11,558(100.0)$ & $<0.001$ \\
\hline Non-drinker & $2,889(22.1)$ & $1,442(12.5)$ & \\
\hline Mild to moderate drinker & $9,984(76.2)$ & $8,507(73.6)$ & \\
\hline Heavy drinker & $221(1.7)$ & $1,609(13.9)$ & \\
\hline $\operatorname{AUDIT}(\mathrm{N}=24,618)$ & $13,081(100.0)$ & $11,537(100.0)$ & $<0.001$ \\
\hline 0-7 (Zone 1) & $11,281(86.2)$ & $5,653(49.0)$ & \\
\hline 8-15 (Zone 2) & $1,372(10.5)$ & $3,617(31.4)$ & \\
\hline $16-19$ (Zone 3$)$ & $222(1.7)$ & $1,155(10.0)$ & \\
\hline$\geq 20$ (Zone 4$)$ & $206(1.6)$ & $1,112(9.6)$ & \\
\hline High-risk drinking $(\mathrm{N}=29,160)$ & $16,786(100.0)$ & $12,374(100.0)$ & $<0.001$ \\
\hline Yes & $636(4.8)$ & $2,309(21.0)$ & \\
\hline No & $16,150(95.2)$ & $10,065(79.0)$ & \\
\hline Smoking status $(\mathrm{N}=28,614)$ & $16,437(100.0)$ & $12,177(100.0)$ & $<0.001$ \\
\hline Never & $14,657(89.2)$ & $2,415(19.8)$ & \\
\hline Ex-former & $889(5.4)$ & 4,949 (40.7) & \\
\hline Current & $891(5.4)$ & $4,813(39.5)$ & \\
\hline Regular exercise $(\mathrm{N}=28,256)$ & $16,262(100.0)$ & $11,994(100.0)$ & $<0.001$ \\
\hline Yes & $2,731(16.8)$ & $2,776(23.1)$ & \\
\hline No & $13,531(83.2)$ & 9,218 (76.9) & \\
\hline Sleep duration $(\mathrm{N}=28,565)$ & $9.2 \pm 0.2$ & $8.5 \pm 0.2$ & $<0.001$ \\
\hline Presence of psychiatric or medical diseases $(\mathrm{N}=29,158)$ & $16,786(100.0)$ & $12,372(100.0)$ & $<0.001$ \\
\hline Yes & $6,459(38.5)$ & $4,314(34.9)$ & \\
\hline No & $10,327(61.5)$ & $8,058(65.1)$ & \\
\hline
\end{tabular}

Values are presented as number (\%) or mean \pm standard errors. Psychiatric or medical diseases: depression, hypertension, dyslipidemia, stroke, myocardiac infarction, angina, osteoarthritis, rheumatoid arthritis, pulmonary tuberculosis, asthma, chronic renal failure, atopic dermatitis, diabetes, thyroid diseases, hepatitis, liver cirrhosis, and stomach, liver, colon, breast, cervix, lung, thyroid, and other cancers 
mood.

Suicidal ideation was assessed by participants' positive answer to the following question: "During the past year, have you ever felt that you were willing to die?" A "yes" or "no" response was also used to determine whether the participants have suicidal thoughts. This item is a well-documented predictor of suicidal attempt that has been previously used in other surveys. ${ }^{22}$

\section{Ethical issues}

The Institutional Review Board (IRB) at the Korea Centers for Disease Control and Prevention approved the study protocol (IRB number: 2010-02CON-21-C, 2011-02CON-06-C, 2012-01EXP-01-2C, 2013-07CON-03-4C, and 2015-01-02$6 \mathrm{C}$ ), and written informed consent was provided by all the participants. In addition, this study was approved by the IRB of Seoul St. Mary's Hospital (IRB number: KC18ZESI0573) to analyze KNHANES data.

\section{Statistical analysis}

Statistical analyses were performed using SPSS version 24.0 (IBM Corp., Armonk, NY, USA) to reflect the complex sampling design. All sampling and weight variables were stratified, and survey sample weights were used for all the analyses to produce non-biased estimates for the descriptive and analytic data analyses. The weighted value was created for a fiveyear-period. Estimates were not provided for the category of missing values. For all analyses, we used a list-wise deletion method to handle the missing values.

Differences in sociodemographic and health-related data including psychological distress between males and females were tested using the chi-square test or independent $\mathrm{t}$-test. All categorical variables are presented as numbers with percentages, and all continuous variables as means with standard errors (SEs).

Multiple logistic regression analyses were used to assess the associations between the drinking or AUDIT levels and psy- chological distress. The odds ratios (ORs) and 95\% confidence intervals (95\% CIs) were also estimated after adjusting for potential confounders. For analyzing the associations of drinking levels with psychological distress, sex, age, education level, marital status, employment status, household income, smoking status, physical activity status, sleep duration, presence of medical or psychiatric conditions, high-risk drinking, and AUDIT levels were adjusted, and the drinking levels instead of AUDIT levels with above factors were adjusted in the analysis of the associations of AUDIT levels with psychological distress.

To evaluate the gender differences in the associations between the drinking or AUDIT levels and psychological distress, all the above variables except sex were adjusted, and menstrual condition (presence or absence of menstruation) was additionally adjusted for women. The p-values were twotailed and those less than 0.05 were considered statistically significant.

\section{RESULTS}

The demographic characteristics of the genders are summarized in Table 1 . The mean age was $46.8 \pm 0.2$ and $44.8 \pm 0.2$ years in females and males respectively, and women were older than men. Females were less likely than males to have a college degree, occupation, higher household income, and absence of medical or psychiatric conditions. In addition, more men than women were married, current smokers, and engaging in regular exercise. Sleep duration was longer in women. The percentage of heavy drinkers (1.7\% vs. $13.9 \%$ ) and possible alcohol dependence (Zone 4 ) (1.6\% vs. $9.6 \%$ ) was lower in females than males.

Table 2 presents gender differences in psychological distress. The numbers of individuals with moderate to severe stress perception (28.0\% vs. $23.1 \%$ ), experience of depressive mood ( $16.3 \%$ vs. $9.0 \%$ ), and having suicidal ideation ( $13.5 \%$ vs. $7.9 \%)$

Table 2. Psychological distress in female and male samples

\begin{tabular}{|c|c|c|c|}
\hline Variables & Female & Male & $\mathrm{p}$ value \\
\hline Degree of stress perception $(\mathrm{N}=28,611)$ & $16,432(100.0)$ & $12,179(100.0)$ & $<0.001$ \\
\hline Moderate to severe & $4,597(28.0)$ & $2,808(23.1)$ & \\
\hline None to mild & $11,835(72.0)$ & $9,371(76.9)$ & \\
\hline Experience of depressive mood for two or more continuous weeks $(\mathrm{N}=28,615)$ & $16,432(100.0)$ & $12,180(100.0)$ & $<0.001$ \\
\hline Yes & $2,674(16.3)$ & $1,094(9.0)$ & \\
\hline No & $13,761(83.7)$ & $11,086(91.0)$ & \\
\hline Suicidal ideation during the previous year $(\mathrm{N}=28,614)$ & $16,436(100.0)$ & $12,178(100.0)$ & $<0.001$ \\
\hline Yes & $2,225(13.5)$ & 964 (7.9) & \\
\hline No & $14,211(86.5)$ & $11,214(92.1)$ & \\
\hline
\end{tabular}

Values are presented as number (\%) 


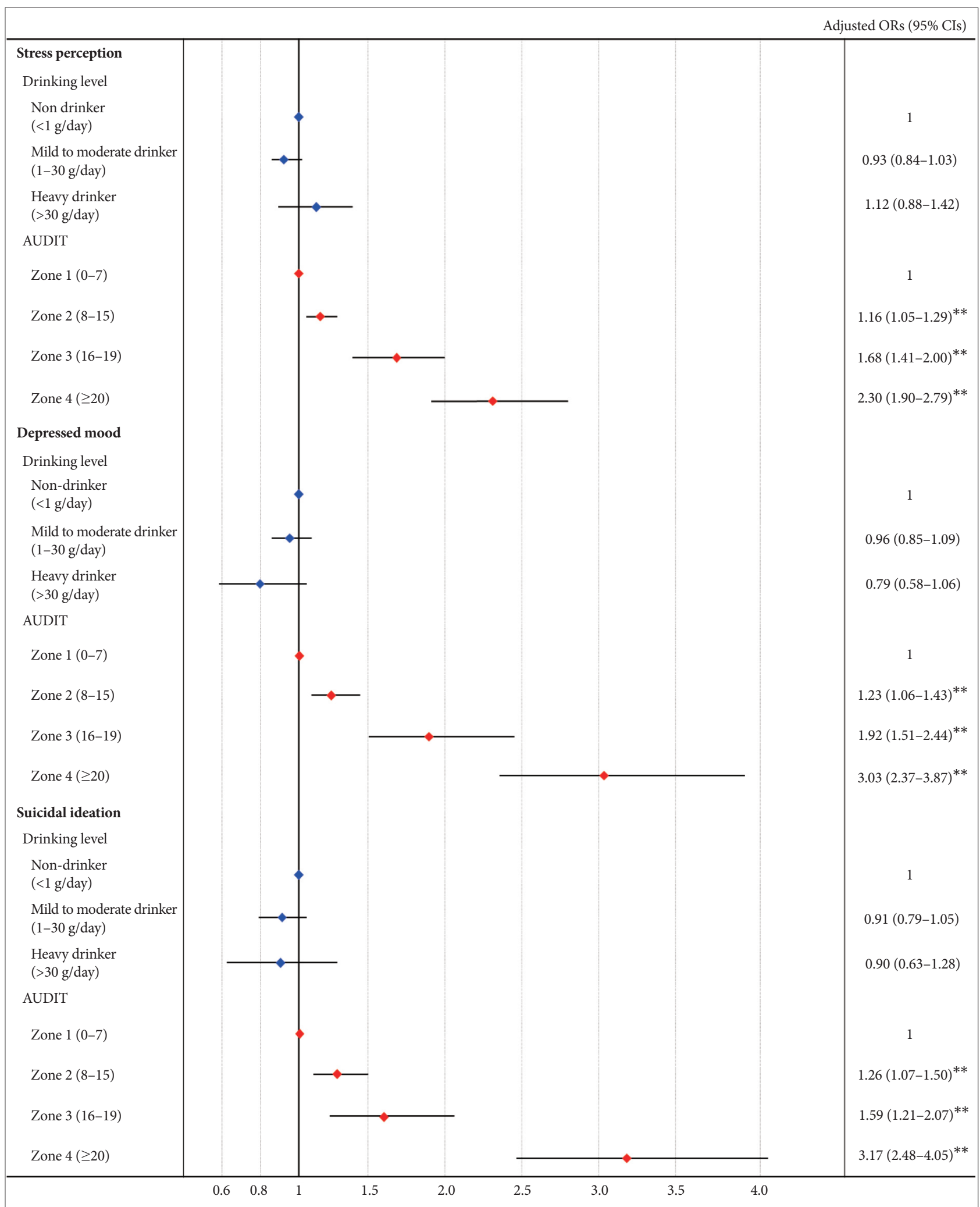

Figure 2. Adjusted odds ratios of psychological distress (stress perception, depressed mood, and suicidal ideation) in whole participants for drinking and AUDIT levels. Multiple logistic analysis, adjusted by age, education, occupation, household income, marital status, high-risk drinking, AUDIT levels, smoking status, regular exercise, sleep duration, and presence of psychiatric or medical diseases for drinking levels. Multiple logistic analysis, adjusted by age, education, occupation, household income, marital status, high-risk drinking, drinking levels, smoking status, regular exercise, sleep duration, and presence of psychiatric or medical diseases for AUDIT levels. ${ }^{* *} p<0.01$. AUDIT: alcohol use disorders Identification Test, OR: odds ratio, Cl: confidence interval. 
were significantly higher in females than males.

Figure 2 shows adjusted ORs and their 95\% CIs of psychological distress from multiple logistic regression analyses in whole participants for drinking and AUDIT levels, respectively. The risks of psychological distress were not significantly associated with drinking levels after adjusting for confounding variables. According to AUDIT levels, however, the risk of psychological distress increased as the score increased based on Zone 1 (AUDIT scores: 0-7) after adjustment. The risks of stress perception in Zones 2, 3, and 4 were 1.16, 1.68, and 2.30 times higher than Zone 1, respectively. The risks of depressed mood were 1.23, 1.92, and 3.03 times higher, and suicidal ideation were $1.26,1.59$, and 3.17 times higher, respectively.

Figure 3 presents the gender differences in the risks of psychological distress according to the drinking and AUDIT levels. The risks of stress perception, depressed mood, and suicidal ideation were not significantly associated with drinking levels after adjusting for confounding variables in both females and males. By contrast, the risks for psychological distress according to AUDIT levels showed a difference between men and women. With regard to stress perception, the risks were significantly high in Zone 3 (adjusted ORs 1.49 in females and 1.67 in males) and 4 (adjusted ORs 2.46 in females and 2.25 in males) and not Zone 2 in both men and women. The risk in Zone 3 was higher in males than in females, whereas in Zone 4 , the risk was higher in females than in males. The risks of depressed mood were high under conditions of Zones 3 and 4 (adjusted ORs 1.72 and 2.80, respectively) in males, while the risks were high even under condition of Zone 2 (adjusted OR 1.42) as well as Zones 3 and 4 (adjusted ORs 2.31 and 3.45, respectively) in females. In addition, the risks for depressed mood in Zones 3 and 4 were higher in women than in men. In terms of suicidal ideation, the risks were high even in Zone 2 (adjusted OR 1.53) as well as Zones 3 and 4 (adjusted ORs 1.90 and 3.48 , respectively) in women, whereas the risk was 2.88 times higher only in Zone 4 in men.

\section{DISCUSSION}

The present study demonstrated that the risks of psychological distress were associated with an increase in AUDIT levels and there were gender differences in the psychological consequences of alcohol-related problems in the nationally representative sample.

In Zone 4 of AUDIT, the risk of stress perception was 2.30 times higher, the risk for depressed mood was 3.03 times higher, and the risk for suicidal ideation was 3.17 times higher than that of Zone 1. In addition, the risks for psychological distress were also higher in Zones 2 and 3 than in Zone 1. The present findings about the association between psychological distress and AUDIT levels broadly support the work of previous other studies in the area linking AUD and psychiatric symptoms. In humans and rodents, chronic alcohol consumption results in a general elevation in blood corticosteroid levels and also alters corticotropin-releasing factor activity independent from the hypothalamic-pituitary-adrenal (HPA) axis. ${ }^{23-29}$ In addition, several prospective and longitudinal studies have shown that problematic alcohol drinking is significantly associated with depressive symptoms and that the causal role of problematic alcohol consumption was stronger than the causal role of greater levels of depressed mood. ${ }^{30-32}$ However, the relationship between alcohol consumption and depressed mood is inconclusive regarding the direction of effects. Furthermore, individuals with alcoholism have a 60-120 times greater suicide risk than the non-psychiatrically ill population. ${ }^{8}$

Remarkably, the association between problematic drinking and psychological distress is more drastic in women than men. In addition, women showed a significant association even though the severity of drinking problem was low with the exception of stress perception. These findings support the presence of gender differences in alcohol-related psychological problems and the higher susceptibility of women to the negative effects of alcohol on psychological health. According to previous findings, these gender differences in alcohol's detrimental effects may be driven by gender differences in alcohol pharmacokinetics and neurotransmitter systems and the influence of gonadal steroid hormones on them. Regarding pharmacokinetics, evidence suggests that women have a higher blood alcohol concentration after consuming equivalent amounts of alcohol than men because women have a lower proportion of body water than men of similar body weight. ${ }^{33-35}$ In addition, the sex disparity in gastric mucosa alcohol dehydrogenase $(\mathrm{ADH})$ activity, which is involved in the first-pass ethanol metabolism, may likely contribute to the blood level of alcohol and alcoholic complications following alcohol intake. ${ }^{36-38}$ Some reports suggested that total $\mathrm{ADH}$ activities including in stomach and liver were significantly higher in males than females, ${ }^{12,39}$ while others did not. ${ }^{40}$ Therefore, these results imply that longer persistence of high ethanol blood level exists in women compared to men after alcohol intake, and thus, women tend to be exposed to higher degrees of alcohol in organs such as the brain that can affect mood and suicidal ideation. Regarding neurotransmitter system and the influence of gonadal hormones, males and females show differential patterns of neurotransmitter release and receptor availability in response to alcohol consumption. ${ }^{12}$ Alcohol is known to alter $\gamma$-aminobutyric acid (GABA) system through the GABAA receptor $^{41,42}$ and other neurotransmitter systems such as the serotonergic system, ${ }^{11}$ and sex is an important factor for neurotransmitters' receptor subunit expression. ${ }^{43-45}$ In addition, fe- 
male gonadal hormones affect many neurobiological responses, ${ }^{11}$ and specifically, changes in estrogen levels have been shown to influence concentrations of serotonin and serotonin-recep- tor subtype. ${ }^{46}$ This interaction may contribute to an increased vulnerability to depression or suicidal ideation in women.

However, gender differences in the association between
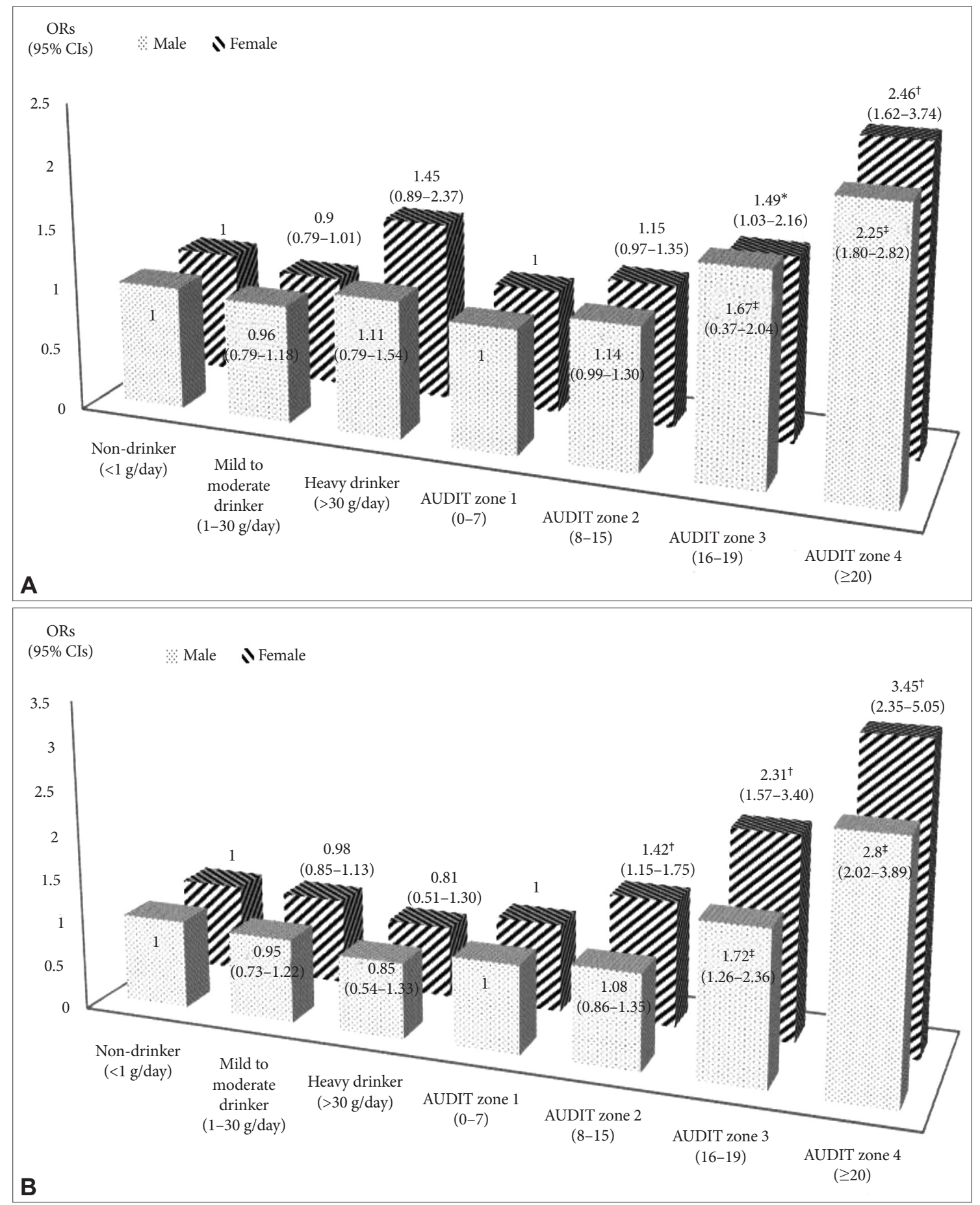

Figure 3. Adjusted odds ratios of psychological distress for drinking and AUDIT levels between genders. A: stress perception, B: depressed mood, C: suicidal ideation. Multiple logistic analysis, adjusted by age, education, occupation, household income, marital status, high-risk drinking, AUDIT levels, smoking status, regular exercise, sleep duration, and presence of psychiatric or medical diseases for drinking levels. Multiple logistic analysis, adjusted by age, education, occupation, household income, marital status, high-risk drinking, drinking levels, smoking status, regular exercise, sleep duration, and presence of psychiatric or medical diseases for AUDIT levels. Additionally adjusted menstrual condition (presence or absence of menstruation) in females. ${ }^{*} p<0.05,{ }^{\dagger} p<0.01$ in females, ${ }^{\ddagger} p<0.01$ in males. AUDIT: Alcohol use Disorders Identification Test, OR: odds ratio, $\mathrm{Cl}$ : confidence interval. 


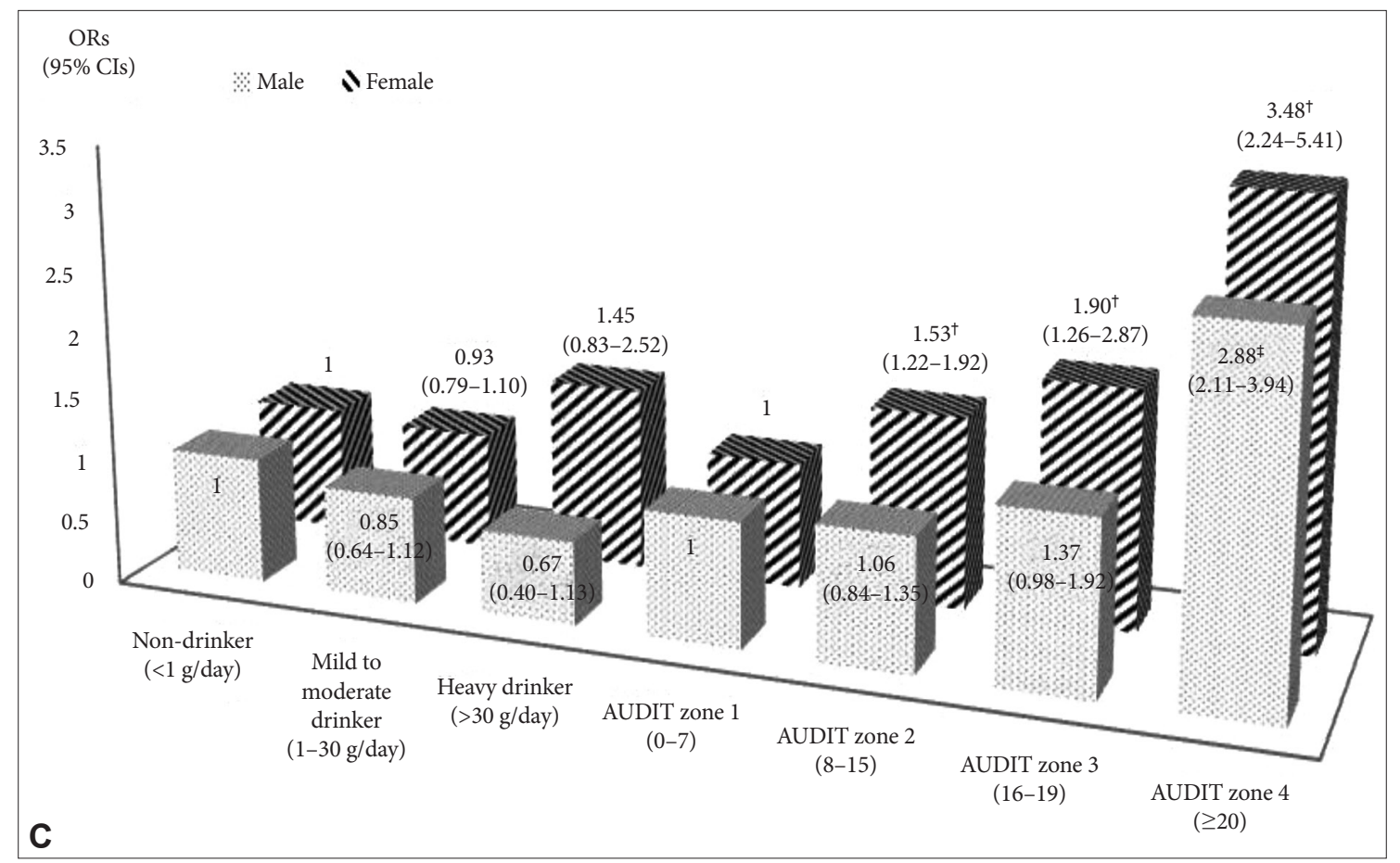

Figure 3. (continued) Adjusted odds ratios of psychological distress for drinking and AUDIT levels between genders. A: stress perception, B: depressed mood, C: suicidal ideation. Multiple logistic analysis, adjusted by age, education, occupation, household income, marital status, high-risk drinking, AUDIT levels, smoking status, regular exercise, sleep duration, and presence of psychiatric or medical diseases for drinking levels. Multiple logistic analysis, adjusted by age, education, occupation, household income, marital status, high-risk drinking, drinking levels, smoking status, regular exercise, sleep duration, and presence of psychiatric or medical diseases for AUDIT levels. Additionally adjusted menstrual condition (presence or absence of menstruation) in females. ${ }^{*} p<0.05,{ }^{\dagger} p<0.01$ in females, ${ }^{\ddagger} p<0.01$ in males. AUDIT: Alcohol use Disorders Identification Test, OR: odds ratio, $\mathrm{Cl}$ : confidence interval.

problematic drinking and stress perception showed a different pattern from depressed mood or suicidal ideation. In both men and women, Zones 3 and 4 showed higher stress perception than Zone 1, and in Zone 3, the risk for stress perception was higher in men than in women. So far, the complex relationship between alcohol and stress is poorly understood. Alcohol is known to be an effective anxiolytic, ${ }^{47-49}$ whereas alcohol can serve as a stressor that stimulates the HPA axis, with the magnitude and response degree being influenced by gender or alcohol drinking level. ${ }^{50}$ Alcohol also alters stress reactivity differentially among men and women..$^{51}$ Previous studies have indicated that men show greater stress-related brain response in the fronto-limbic area ${ }^{52}$ and higher cortisol response, ${ }^{53-55}$ whereas women show blunted adrenal responsivity ${ }^{56}$ and greater tendencies to negative cognitions such as rumination under stress. ${ }^{57}$ On the other hand, some studies have reported that alcohol consumption predicted decreased stress the day following alcohol intake in both sexes. ${ }^{51}$ In addition, several factors such as individual differences in stress appraisal, coping strategies to stress, and response to alcohol intoxication play a role in modulating alcohol-stress interaction. ${ }^{58-60}$ As such, the alcohol, stress, and gender interaction is complex and dynamic, and future studies with valid animal models are needed to understand mechanisms that underlie the complexity of alcohol and stress in males and females.

It is interesting that the present study did not find a significant association between drinking level and psychological distress in both males and females contrary to our expectation. Investigators found that a direct correlation between daily amounts of alcohol intake and increased risk for cancer or a curvilinear effect for coronary heart disease. ${ }^{3}$ However, the findings of the current study do not support the previous research. This discrepancy suggests that the association between alcohol and psychological distress is not simply explained by the amount of alcohol consumed and can be influenced by other factors. In this context, epidemiological researchers have suggested beyond measures of "quantity consumed" to examine the importance of drinking patterns or type in explaining the consequences of alcohol on health. Previous studies have shown that specific beverage type (liquor, not beer) consumption was associated with poorer mental health, even in individuals who consume a light to moderate volume of alcohol. ${ }^{61}$ Therefore, comprehensively assessing the drinking pattern and their related behaviors using structured questionnaires such as AUDIT-that are not limited only to the amount of alcohol intake-is required to predict the psychological consequences of alcohol consumption.

The findings of the present study need to be interpreted in 
light of the following limitations. First, psychological distress was assessed using simple self-report measures on the nature of data on national population study and only in the last one year regarding depressed mood and suicidal ideation. Questionnaires such as Patient Health Questionnaire-9, which is an instrument to screen for the presence and severity of depression and included in the 2014 6th KNHANES are more helpful to obtain a more accurate assessment for psychological distress. Second, we did not control other factors including personality factors, negative life events, family history of psychiatric illnesses, or social support that could affect psychological distress, even though we controlled several factors to identify the association between alcohol drinking or problematic drinking and psychological distress. Third, because this was a cross-sectional study, the establishment of cause-and-effect relationships between alcohol and psychological distress remains limited. Although multiple regression analysis was used to infer the causal relationship, these inferences can be further supported through animal and prospective and longitudinal human studies.

Despite these limitations, the primary strength of the present study is that all the data were obtained from a nationwide population study with a high response rate and sufficient sample size, and they provide representative information regarding the general Korean population. Furthermore, the findings have important implications for clinical practice such as conveying that comprehensive assessment like AUDIT is needed to evaluate the alcohol effect on psychological distress and that individuals-particularly women-who are even at low-risk for problematic drinking should be screened for psychological distress issues.

Approaches to assess psychological distress should take sex disparity into consideration. It is recommended that women with even low-level problematic drinking should be assessed for psychological symptoms including sub-threshold level, while men who have moderate to high-level problematic drinking should be assessed, particularly for the degree of stress level. Furthermore, further investigation focused on the mechanisms of alcohol effect on psychological distress and future studies with consideration for known and emerging genderspecific factors are needed to develop more appropriate evaluation and management strategies for both men and women.

\section{Acknowledgments}

This research was supported by Research Fund of Seoul St. Mary's Hospital, The Catholic University of Korea.

\section{Conflicts of Interest}

The authors have no potential conflicts of interest to disclose.

\section{Author Contributions}

Jo-Eun Jeong, Soo-Hyun Joo, Changtae Hahn, and Tae-Suk Kim designed the study. Jo-Eun Jeong led data analysis and the writing of the manuscript.
Dai-Jin Kim and Tae-Suk Kim supervised the interpretation of the results.

\section{ORCID iDs}

Tae-Suk Kim

https://orcid.org/0000-0002-2488-2904

Jo-Eun Jeong https://orcid.org/0000-0002-4200-278X

\section{REFERENCES}

1. World Health Organization. Global Status Report on Alcohol and Health 2014. Geneva: WHO Press; 2014.

2. Korea Health Statistics 2016: Korea National Health and Nutrition Examination Survey (KNHANES VII-1). Available at: https://knhanes. cdc.go.kr/knhanes/sub04/sub04_03.do?classType=7. Accessed October 30, 2018.

3. Mannelli P, Pae CU. Medical comorbidity and alcohol dependence. Curr Psychiatry Rep 2007;9:217-224.

4. Mukherjee S. Alcoholism and its effects on the central nervous system. Curr Neurovasc Res 2013;10:256-262.

5. Boden JM, Fergusson DM. Alcohol and depression. Addiction 2011;106: 906-914.

6. Madden JS. Alcohol and depression. Br J Hosp Med 1993;50:261-264.

7. Becker HC, Lopez MF, Doremus-Fitzwater TL. Effects of stress on alcohol drinking: a review of animal studies. Psychopharmacology (Berl) 2011;218:131-156.

8. Sher L. Alcohol and suicide: neurobiological and clinical aspects. ScientificWorldJournal 2006;6:700-706.

9. Rehm J, Room R, Graham K, Monteiro M, Gmel G, Sempos CT. The relationship of average volume of alcohol consumption and patterns of drinking to burden of disease: an overview. Addiction 2003;98:1209-1228.

10. Rehm J, Kanteres F, Lachenmeier DW. Unrecorded consumption, quality of alcohol and health consequences. Drug Alcohol Rev 2010;29:426436.

11. Ceylan-Isik AF, McBride SM, Ren J. Sex difference in alcoholism: who is at a greater risk for development of alcoholic complication? Life Sci 2010;87:133-138.

12. Erol A, Karpyak VM. Sex and gender-related differences in alcohol use and its consequences: Contemporary knowledge and future research considerations. Drug Alcohol Depend 2015;156:1-13.

13. Becker JB, Perry AN, Westenbroek C. Sex differences in the neural mechanisms mediating addiction: a new synthesis and hypothesis. Biol Sex Differ 2012;3:14.

14. Nolen-Hoeksema S. Gender differences in risk factors and consequences for alcohol use and problems. Clin Psychol Rev 2004;24:981-1010.

15. Cyders MA, VanderVeen JD, Plawecki M, Millward JB, Hays J, Kareken DA, et al. Gender-Specific Effects of Mood on Alcohol-Seeking Behaviors: Preliminary Findings Using Intravenous Alcohol Self-Administration. Alcohol Clin Exp Res 2016;40:393-400.

16. Dufour MC. What is moderate drinking? Defining "drinks" and drinking levels. Alcohol Res Health 1999;23:5-14.

17. Meneses-Gaya Cd, Zuardi AW, Loureiro SR, Crippa JAS. Alcohol Use Disorders Identification Test (AUDIT): an updated systematic review of psychometric properties. Psychol Neurosci 2009;2:83-97.

18. Saunders JB, Aasland OG, Babor TF, de la Fuente JR, Grant M. Development of the Alcohol Use Disorders Identification Test (AUDIT): WHO Collaborative Project on Early Detection of Persons with Harmful Alcohol Consumption--II. Addiction 1993;88:791-804.

19. Donovan DM, Kivlahan DR, Doyle SR, Longabaugh R, Greenfield SF. Concurrent validity of the Alcohol Use Disorders Identification Test (AUDIT) and AUDIT zones in defining levels of severity among outpatients with alcohol dependence in the COMBINE study. Addiction 2006;101:1696-1704.

20. Chung W, Lim S, Lee S. Why is high-risk drinking more prevalent among men than women? Evidence from South Korea. BMC Public Health 2012; 12:101.

21. Gigantesco A, Morosini P. Development, reliability and factor analysis 
of a self-administered questionnaire which originates from the World Health Organization's Composite International Diagnostic Interview Short Form (CIDI-SF) for assessing mental disorders. Clin Pract Epidemiol Ment Health 2008;4:8.

22. Gaynes BN, West SL, Ford CA, Frame P, Klein J, Lohr KN. Screening for suicide risk in adults: a summary of the evidence for the U.S. Preventive Services Task Force. Ann Intern Med 2004;140:822-835.

23. Errico AL, Parsons OA, King AC, Lovallo WR. Attenuated cortisol response to biobehavioral stressors in sober alcoholics. J Stud Alcohol 1993; 54:393-398.

24. Kakihana R, Moore JA. Circadian rhythm of corticosterone in mice: the effect of chronic consumption of alcohol. Psychopharmacologia 1976;46: 301-305.

25. Lee S, Schmidt D, Tilders F, Cole M, Smith A, Rivier C. Prolonged exposure to intermittent alcohol vapors blunts hypothalamic responsiveness to immune and non-immune signals. Alcohol Clin Exp Res 2000;24:110122.

26. Rasmussen DD, Boldt BM, Bryant CA, Mitton DR, Larsen SA, Wilkinson CW. Chronic daily ethanol and withdrawal: 1 . Long-term changes in the hypothalamo-pituitary-adrenal axis. Alcohol Clin Exp Res 2000; 24:1836-1849.

27. Heilig M, Koob GF. A key role for corticotropin-releasing factor in alcohol dependence. Trends Neurosci 2007;30:399-406.

28. Koob GF, Zorrilla EP. Neurobiological mechanisms of addiction: focus on corticotropin-releasing factor. Curr Opin Investig Drugs 2010;11:6371.

29. Uhart M, Wand GS. Stress, alcohol and drug interaction: an update of human research. Addict Biol 2009;14:43-64.

30. Paljarvi T, Koskenvuo M, Poikolainen K, Kauhanen J, Sillanmaki L, Makela P. Binge drinking and depressive symptoms: a 5-year population-based cohort study. Addiction 2009;104:1168-1178.

31. Flensborg-Madsen T, Mortensen EL, Knop J, Becker U, Sher L, Gronbaek M. Comorbidity and temporal ordering of alcohol use disorders and other psychiatric disorders: results from a Danish register-based study. Compr Psychiatry 2009;50:307-314.

32. Fergusson DM, Boden JM, Horwood LJ. Tests of causal links between alcohol abuse or dependence and major depression. Arch Gen Psychiatry 2009;66:260-266.

33. Ammon E, Schafer C, Hofmann U, Klotz U. Disposition and first-pass metabolism of ethanol in humans: is it gastric or hepatic and does it depend on gender? Clin Pharmacol Ther 1996;59:503-513.

34. Ely M, Hardy R, Longford NT, Wadsworth ME. Gender differences in the relationship between alcohol consumption and drink problems are largely accounted for by body water. Alcohol Alcohol 1999;34:894-902.

35. Sutker PB, Tabakoff B, Goist KC Jr, Randall CL. Acute alcohol intoxication, mood states and alcohol metabolism in women and men. Pharmacol Biochem Behav 1983;18 (Suppl 1):349-354.

36. Baraona E, Abittan CS, Dohmen K, Moretti M, Pozzato G, Chayes ZW, et al. Gender differences in pharmacokinetics of alcohol. Alcohol Clin Exp Res 2001;25:502-507.

37. Frezza M, di Padova C, Pozzato G, Terpin M, Baraona E, Lieber CS. High blood alcohol levels in women. The role of decreased gastric alcohol dehydrogenase activity and first-pass metabolism. N Engl J Med 1990;322: 95-99.

38. Ward RJ, Coutelle C. Women and alcohol susceptibility: could differences in alcohol metabolism predispose women to alcohol-related diseases? Arch Womens Ment Health 2003;6:231-238.

39. Chrostek L, Jelski W, Szmitkowski M, Puchalski Z. Gender-related differences in hepatic activity of alcohol dehydrogenase isoenzymes and aldehyde dehydrogenase in humans. J Clin Lab Anal 2003;17:93-96.

40. Yin SJ, Liao CS, Wu CW, Li TT, Chen LL, Lai CL, et al. Human stomach alcohol and aldehyde dehydrogenases: comparison of expression pattern and activities in alimentary tract. Gastroenterology 1997;112:766-775.

41. Abramov U, Raud S, Innos J, Koks S, Matsui T, Vasar E. Gender specific effects of ethanol in mice, lacking CCK2 receptors. Behav Brain Res 2006; 175:149-156.

42. Grobin AC, Matthews DB, Devaud LL, Morrow AL. The role of GABA(A) receptors in the acute and chronic effects of ethanol. Psychopharmacology (Berl) 1998;139:2-19.

43. Devaud LL, Matthews DB, Morrow AL. Gender impacts behavioral and neurochemical adaptations in ethanol-dependent rats. Pharmacol Biochem Behav 1999;64:841-849.

44. Raud S, Innos J, Abramov U, Reimets A, Koks S, Soosaar A, et al. Targeted invalidation of CCK2 receptor gene induces anxiolytic-like action in light-dark exploration, but not in fear conditioning test. Psychopharmacology (Berl) 2005;181:347-357.

45. Raud S, Runkorg K, Veraksits A, Reimets A, Nelovkov A, Abramov U, et al. Targeted mutation of CCK2 receptor gene modifies the behavioural effects of diazepam in female mice. Psychopharmacology (Berl) 2003;168: 417-425.

46. Kornstein SG, Sloan DM, Thase ME. Gender-specific differences in depression and treatment response. Psychopharmacol Bull 2002;36:99-112.

47. Brady KT, Sonne SC. The role of stress in alcohol use, alcoholism treatment, and relapse. Alcohol Res Health 1999;23:263-271.

48. Pohorecky LA. Stress and alcohol interaction: an update of human research. Alcohol Clin Exp Res 1991;15:438-459.

49. Sayette MA. Does drinking reduce stress? Alcohol Res Health 1999;23: 250-255.

50. Wand G. Review of NIAAA's Neuroscience and Behavioral Research Portfolio: NIAAA Research Monograph. In: Noronha A, Eckardt M, Warren K, Editors. Hypothalamic-Pituitary-Adrenal Axis: Changes and Risk for Alcoholism. Bethesda: NIAAA Press, 2000, p.397-415.

51. Ayer LA, Harder VS, Rose GL, Helzer JE. Drinking and stress: an examination of sex and stressor differences using IVR-based daily data. Drug Alcohol Depend 2011;115:205-212.

52. Goldstein JM, Jerram M, Abbs B, Whitfield-Gabrieli S, Makris N. Sex differences in stress response circuitry activation dependent on female hormonal cycle. J Neurosci 2010;30:431-438.

53. Jackson ED, Payne JD, Nadel L, Jacobs WJ. Stress differentially modulates fear conditioning in healthy men and women. Biol Psychiatry 2006; 59:516-522.

54. Kudielka BM, Kirschbaum C. Sex differences in HPA axis responses to stress: a review. Biol Psychol 2005;69:113-132.

55. Verona E, Reed A 2nd, Curtin JJ, Pole M. Gender differences in emotional and overt/covert aggressive responses to stress. Aggress Behav 2007; 33:261-271.

56. Brady KT, Waldrop AE, McRae AL, Back SE, Saladin ME, Upadhyaya HP, et al. The impact of alcohol dependence and posttraumatic stress disorder on cold pressor task response. J Stud Alcohol 2006;67:700-706.

57. Nolen-Hoeksema S. Sex differences in unipolar depression: evidence and theory. Psychol Bull 1987;101:259-282.

58. Childs E, O'Connor S, de Wit $\mathrm{H}$. Bidirectional interactions between acute psychosocial stress and acute intravenous alcohol in healthy men. Alcohol Clin Exp Res 2011;35:1794-1803.

59. Claessens SE, Daskalakis NP, van der Veen R, Oitzl MS, de Kloet ER, Champagne DL. Development of individual differences in stress responsiveness: an overview of factors mediating the outcome of early life experiences. Psychopharmacology (Berl) 2011;214:141-154.

60. Williams PG, Suchy Y, Rau HK. Individual differences in executive functioning: implications for stress regulation. Ann Behav Med 2009;37:126140.

61. Stranges S, Notaro J, Freudenheim JL, Calogero RM, Muti P, Farinaro E, et al. Alcohol drinking pattern and subjective health in a populationbased study. Addiction 2006;101:1265-1276. 